\title{
Effect of Inclusion of Electron Correlation in MM3 Studies of Cyclic Conjugated Compounds
}

\author{
JULIA C. TAI, ${ }^{1}$ NORMAN L. ALLINGER ${ }^{2}$ \\ ${ }^{1}$ Department of Natural Sciences, University of Michigan-Dearborn, Dearborn, Michigan 48128-1491 \\ ${ }^{2}$ Computational Center for Molecular Structure and Design, Department of Chemistry, University of \\ Georgia, Athens, Georgia 30602-2556
}

Received 1 August 1997; accepted 17 September 1997

\begin{abstract}
Electron correlation at the Møller-Plesset second-order level was incorporated into the $\pi$-system portion of MM3 calculations for several conformers of [10]annulene, [18]annulene, bicyclo[5.3.1] undecapentaene, and bicyclo[4.4.1] undecapentaene. The conformers with "localized" C $-\mathrm{C} \pi$ bonds (strongly alternating bond lengths) were found to be of lower energy than their counterparts with "delocalized" C $-\mathrm{C} \pi$ bonds (similar bond lengths) before correlation energy was included. Correlation always lowered the energies of the delocalized conformation more than it did that of the localized conformation, such that often the latter was found to be more stable after correlation energy was included in the calculation. When a delocalized structure was not at a stationary point on the MM3 energy surface, such comparison could not be made. An example is the porphin molecule. (c) 1998 John Wiley \& Sons, Inc. J Comput Chem 19: 475-487, 1998
\end{abstract}

Keywords: molecular mechanics; correlation energies; conjugated polyenes

\section{Introduction}

$\mathbf{T}$ he molecular mechanics force fields MM2 and MM3 have been very successful in predicting the structures, energies, and related properties of a variety of compounds. ${ }^{1}$ However, in a

Correspondence to: N. L. Allinger; e-mail: allinger@ sunchem.chem.uga.edu

Contract/grant sponsor: Petroleum Res. Fund-ACS few cases among the conjugated ring compounds where either a structure with alternate long and short bond lengths (butadiene type) or naphthalenelike bond lengths (with many bonds of nearly benzene bond length, possibly interspersed by bonds of different length) could exist, MM2 or MM3 usually predicts the one with alternate bond lengths to be of lower energy, while experiments seem to sometimes favor the other. Some of the compounds in question are [18]annulene, bicyclo[4.4.1] undecapentaene, bicyclo[5.3.1]unde- 
capentaene, and porphin. The structure with alternate bond lengths is sometimes referred to as $\pi$-bond localized, and the one with naphthalenelike bond lengths is termed as $\pi$-bond delocalized. It had long been suspected and later demonstrated that the Hartree-Fock approximation tends to favor the localized over the delocalized structure. When Baumann $^{2}$ studied the energies of $[n]$ annulenes (where $n=6,8,10,12,14,16$, and 18) at the CNDO level, he found that, except when $n=6$, the localized structure was calculated to have a lower energy than the delocalized one at the Hartree-Fock level. When doubly excited configurations were included in the calculation, he found that the magnitude of the correlation energy was smaller (less negative) for the localized structure than for the delocalized structure. In the case of the larger $[4 n+2]$ annulenes, the correlation energy difference was large enough so that the delocalized structure was predicted to be more stable. The correlation effect was not as important for the $[4 n]$ annulenes. In an $a b$ initio study including geometry optimization for the free base porphin, Almlö et al. $^{3}$ found that at various Hartree-Fock levels, the energy of the more delocalized $D_{2 h}$ form was higher than that of the more localized $\mathrm{C}_{2 \mathrm{v}}$ form, but when electron correlation was included at the Møller-Plesset second-order (MP2) or local density functional (LDF) levels, the $\mathrm{D}_{2 \mathrm{~h}}$ form became the more stable one. In another $a b$ initio study, Xie and colleagues ${ }^{4}$ calculated the energies of several low-lying structural isomers of [10]annulene. They found that inclusion of correlation favored the delocalized structure and altered the relative energies of these isomers from what was predicted by calculations without correlation. More recently, Yoshizawa et $a .^{5}$ studied the relative energies of the $D_{3 h}$ and $D_{6 h}$ structures in [n]annulenes (where $n=6(2 m+1)$, with $m=$ 1-5), using the MNDOC ( $C$ for correlation) method, coupled with a conventional perturbation treatment. They found that the $D_{6 h}$ structures had nearly equal $\mathrm{C}-\mathrm{C}$ bond lengths of approximately $1.4 \AA$, the $D_{3 h}$ ones exhibited strong bond length alternation, and the correlation energy was larger (more negative) in the $\mathrm{D}_{6 \mathrm{~h}}$ due to the small HOMO-LUMO gaps in the $D_{6 h}$ structures. Because MM2 and MM3 do not take electron correlation into account in the treatment of $\pi$ systems, we could not expect them to always correctly predict the relative energies of compounds where both a $\pi$-bond localized and delocalized structure might exist. In the present work, we studied the effect of including correlation within the framework of
MM3 on the relative energies of these structural isomers. We also investigated circumstances under which correct structures might be identified.

\section{Method}

There is no doubt that the inclusion of electron correlation will lead overall to better calculated results, here and elsewhere. The problem is that accurate correlation calculations are very time consuming and inappropriate for inclusion in molecular mechanics. The question is, can an approximate correlation calculation be carried out within the context of molecular mechanics that will be both fast enough and accurate enough to be useful? It was decided that $\pi$-system calculations at the MP2 level would be most appropriate for our purposes. Necessary computer subroutines were written and incorporated into the MM3 program. Mathematical equations needed could be derived in the usual way. ${ }^{6}$ The important equations used are presented below.

The wave function $\Phi_{0}$ of the ground state is written as

$$
\Phi_{0}=\Phi_{0}^{(0)}+\Phi_{0}^{(1)}+\Phi_{0}^{(2)},
$$

where $\Phi_{0}^{(0)}$ is the Hartree-Fock wave function and $\Phi_{0}^{(1)}$ and $\Phi_{0}^{(2)}$ are linear combinations of excited state Hartree-Fock wave functions $\Phi_{n}^{(0)}$. Thus,

$$
\Phi_{0}^{(1)}=\sum C_{n}^{(1)} \Phi_{n}^{(0)} \quad \text { and } \quad \Phi_{0}^{(2)}=\sum C_{n}^{(2)} \Phi_{n}^{(0)},
$$

where

$$
\begin{gathered}
C_{n}^{(1)}=\langle n|V| 0\rangle /\left[E_{0}^{(0)}-E_{n}^{(0)}\right], \\
C_{n}^{(2)}=\left[\sum C_{m}^{(1)}\langle n|V| m\rangle-C_{n}^{(1)}\langle 0|V| 0\rangle\right] / \\
{\left[E_{0}^{(0)}-E_{n}^{(0)}\right] \quad m \neq 0 .}
\end{gathered}
$$

In the above equations, $E_{n}^{(0)}$ is the sum of the orbital energies for state $n$, and $\langle n|V| n\rangle$ equals $E_{n}(\mathrm{HF})-E_{n}^{(0)}$ where $E_{n}(\mathrm{HF})$ is the Hartree-Fock energy for state $n$. The matrix elements $\langle n|V| m\rangle$ are sums of two electron integrals and can be evaluated in the usual way. The ground state energy at the MP2 level, $E_{0}$, is written as $E_{0}=E_{0}^{(0)}$ $+E_{0}^{(1)}+E_{0}^{(2)}+E_{0}^{(3)}$, where $E_{0}^{(0)}+E_{0}^{(1)}$ equals the Hartree-Fock energy and the sum of $E_{0}^{(2)}$ and $E_{0}^{(3)}$ is the electron correlation energy, where

$$
\begin{gathered}
E_{0}^{(2)}=\sum(\langle 0|V| n\rangle)^{2} /\left[E_{0}^{(0)}-E_{n}^{(0)}\right], \quad n \neq 0, \\
E_{0}^{(3)}=\sum C_{n}^{(2)}\langle 0|V| n\rangle .
\end{gathered}
$$


The bond order $p_{\mu \nu}$ for a bond $\mu-\nu$ in the ground state is defined as

$$
p_{\mu \nu}(0)=2 * \sum c_{i \mu} c_{i \nu},
$$

where $c_{i \mu}$ is the coefficient of the $\mu$ th atomic orbital of the $i$ th molecular orbital (MO). The summation is over all occupied MOs in the ground state. We define the bond order in an excited state $i j k l$, where one electron from each of orbitals $i$ and $j$ in the ground state have been promoted to orbitals $k$ and $l$, as

$$
\begin{aligned}
p_{\mu \nu}(i j k l)=p_{\mu \nu}(0)+c_{k \mu} c_{k \nu} & \\
& +c_{l \mu} c_{l \nu}-c_{i \mu} c_{i \nu}-c_{j \mu} c_{j \nu} .
\end{aligned}
$$

When electron correlation is included, the bond order is taken to be the weighted average of the bond order calculated for the ground state and those calculated for the excited states, thus,

$p_{\mu \nu}=N\left[p_{\mu \nu}(0)+\sum\left(C_{n}^{(1)}+C_{n}^{(2)}\right)^{2} p_{\mu \nu}(n)\right] \quad n \neq 0$,

where $N$ is a normalization constant, $N=$ $\left[1+\sum\left(C_{n}^{(1)}+C_{n}^{(2)}\right)^{2}\right]^{-1}$.

Almlöf et al. found when calculating correlation energies of porphin at the MP2 double zeta potential second-order (DZP2) level that correlation descriptions restricted to the $\pi$ electrons missed about half of the correlation stabilization, in agreement with CI results obtained at a $\mathrm{D}_{2 \mathrm{~h}}$ geometry. ${ }^{3}$ Yoshizawa and coworkers ${ }^{5}$ also found in their work with large annulenes that the correlation energy from only the $\pi$ electrons was approximately $40-50 \%$ of the total correlation energy from all valence electrons. The problem facing molecular mechanics is this: any kind of all electron correlation calculation is computationally impractical. How can we approach the problem? Because only the $\pi$ system was included in our calculations, we multiplied the contribution from the excited states (the matrix elements between the ground and doubly excited states) by the square root of 2 so that the correlation energies would be doubled. We termed this calculation MP $2 * 2$. While this may seem like a brutal approximation, each $\pi$ bond in the conjugated system has a corresponding $\sigma$ bond, and the correlation in the two bond components is similar; thus, including the $\sigma$ correlation in this way should be a much better approximation than leaving it out, and such an inclusion is computationally fast.

When electron correlation is included in the molecular mechanics calculation, the $\pi$-bond or- ders change. The bond order of ethylene decreases, for example, while those of benzene increase. Furthermore, the difference between bond orders in a molecule generally decreases. For instance, the bond order of the central $\mathrm{C}-\mathrm{C}$ bond in butadiene increases, while those of the two end bonds decrease. Because the bond order-bond length relationship used in MM3 is based on the calculated bond orders of ethylene, butadiene, and benzene, using the same relationship when electron correlation is included would result in a longer ethylene bond length, longer double bond lengths for butadiene, a shorter single bond length for butadiene, and shorter bond lengths for benzene. A new bond order-bond length relationship could then be derived, based on the bond orders from calculations including electron correlation. Because our purpose here is to obtain the correlation energy, this reparameterization was not carried out. (In fact, the intent is to carry it out when and if the whole procedure proves to be viable.) The structures reported below are the usual MM3 structures without electron correlation. The procedure used here was to do the usual MM3 calculations for the structure and the energy of the molecule first, then the contributions from the doubly excited configurations to the wave function were obtained. The bond order of each $\pi$ bond was then recalculated including these contributions. Geometries were then reoptimized with respect to the steric energy using the new bond orders. The process was repeated until self-consistency, and the correlation energy was noted. However, because a new bond length-bond order relation was not derived and used in this part of the calculation, the structures at this point were not true "MM3" structures; hence, they are not reported. This approach is computationally feasible, and it leads to an increase in computing time of about a factor of 2-5 relative to the Hartree-Fock approximation. Thequestion is, does it lead to an improvement in the results that is sufficient to be useful?

\section{Results}

\section{[10]ANNULENE}

This molecule was studied in some detail to help us to understand the workings of the MM3 minimization procedure for such a system, and the relative importance of electron correlation for different structures. Ordinary MM3 calculations were carried out first. 
We started with a planar $C_{2 h}$ structure and obtained an energy minimized structure using the block-diagonal method. ${ }^{7}$ The structure stayed $C_{2 h}$ planar (1a), even with all symmetry restrictions removed (because it corresponds to a stationary point), and it was then optimized again using the full-matrix method. ${ }^{7}$ However, this structure shows two imaginary vibrational frequencies, which indicates that it is not a local minimum on the potential energy surface. Then we moved the carbon atoms out of the plane: odd numbered atoms in one direction and even numbered in the opposite direction. The corresponding reminimized structure had $\mathrm{C}_{2 \mathrm{~h}}$ nonplanar symmetry (1b). When one of the $1.461 \AA$ bonds in the $C_{2 h}$ planar structure was made shorter and the other longer by moving the carbon atoms closer or farther apart, the minimized structure had $\mathrm{C}_{2}$ (nonplanar) symmetry (1c). Both of these nonplanar structures are true minima on the potential energy surface.

When another starting $\mathrm{C}_{2 \mathrm{~h}}$ (planar) structure was optimized after removing symmetry constraints, a $\mathrm{D}_{2 \mathrm{~h}}$ (planar) structure resulted (1d), which had three imaginary vibration frequencies. The bond lengths of the four structures are shown in Figure 1. The $C_{2}$ structure (1c) has the lowest steric energy, and it is the only one among the four that has alternate long and short $\mathrm{C}-\mathrm{C}$ bonds.

Calculations were then carried out with $\pi$-system electron correlation at the $\mathrm{MP} 2 * 2$ level on

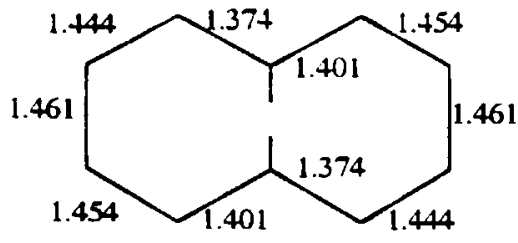

1a. C $_{2 h}$ planar

not an energy minimum structure

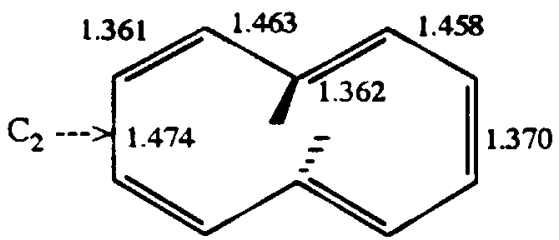

1c. $C_{2}$ nonplanar

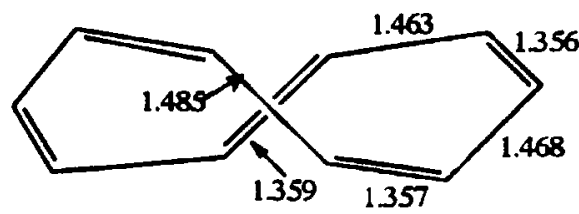

le. $C_{2} M M 3$ bond lengths

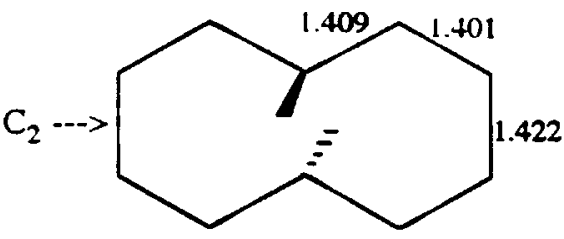

1b. $\mathrm{C}_{2 \mathrm{~h}}$ nonplanar

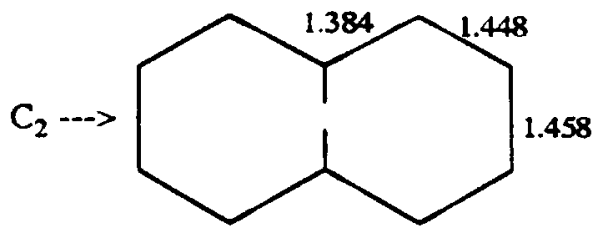

1d. $\mathrm{D}_{2 \mathrm{~h}}$ planar

not an energy minimum structure

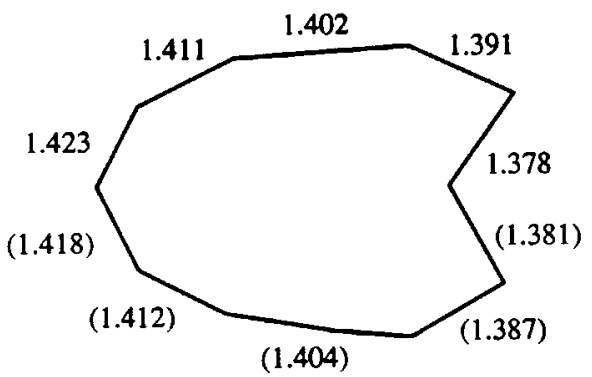

if. $C_{s} B 3 L Y P / T Z P$ and (MM3) bond lengths

FIGURE 1. Structures of [10]annulene. 


\begin{tabular}{|c|c|c|c|c|}
\hline & \multicolumn{4}{|c|}{ Structure } \\
\hline & $\begin{array}{c}\mathrm{C}_{2 \mathrm{~h}} \\
\text { (Planar) }^{\mathrm{a}} \\
1 \mathrm{a}\end{array}$ & $\begin{array}{c}\mathrm{D}_{2 \mathrm{~h}} \\
\text { (Planar) }^{\mathrm{a}} \\
\text { 1d }\end{array}$ & $\begin{array}{c}\mathrm{C}_{2 \mathrm{~h}} \\
\text { (Nonplanar) } \\
\mathbf{1 b}\end{array}$ & $\begin{array}{c}\mathrm{C}_{2} \\
\text { (Nonplanar) } \\
\mathbf{1 c}\end{array}$ \\
\hline Steric energy & 128.4 & 129.1 & 51.3 & 43.9 \\
\hline Total MM3 energy (inc. HF) & -1001.7 & -1001.0 & -1080.7 & -1087.9 \\
\hline Correlation energy MP2 $* 2$ & -48.9 & -48.0 & -43.2 & -35.0 \\
\hline \multicolumn{5}{|l|}{ Relative energy } \\
\hline Without correlation & 86.2 & 86.9 & 7.2 & 0.0 \\
\hline With correlation & 73.3 & 74.9 & 0.0 & 1.0 \\
\hline
\end{tabular}

${ }^{a}$ Not a local minimum energy structure.

each of these four structures. The various energies obtained are listed in Table I. Although structures 1a and $\mathbf{1 d}$ are not true local minima, they are stationary points and are included in the table to show that correlation systematically favors the delocalized structures.

Comparing the two stable structures, $\mathrm{C}_{2 \mathrm{~h}}$ nonplanar and $C_{2}$, we see that correlation lowers the energy of the delocalized $C_{2 h}$ structure more than that of the localized $\mathrm{C}_{2}$ structure. And as a result, the $\mathrm{C}_{2 \mathrm{~h}}$ structure becomes the more stable one at the correlated level. We also see that correlation lowers the energy of the other two delocalized structures $D_{2 h}$ and $C_{2 h}$ (planar) relative to that of the $\mathrm{C}_{2}$ structure. It should be noted that because the geometries were not reparameterized, the correlation energies have only semiquantitative significance.

The first really systematic $a b$ initio study on plausible structures of [10]annulene was reported by Farnell et al. ${ }^{8}$ With single point calculations at the STO-2G, STO-3G, and 4-31G level on STO-2G structures, they found a twisted ring structure of $\mathrm{C}_{2}$ symmetry (1e) to be of the lowest energy, followed by structure 1c (STO-2G) or by an all cis tub structure of symmetry $\mathrm{C}_{\mathrm{s}}$ (STO-3G or 4-31G). The MM3 energy of the twisted structure $1 \mathrm{e}$ is 3.8 and $3.2 \mathrm{kcal}$ higher than that of $\mathbf{1 c}$ before and after correlation energy is added, respectively. The bond lengths of this structure are given in Figure 1e. In a recent article on the structural isomers of [10]annulenes, ${ }^{4}$ Xie et al. reported that while molecular mechanics (MM2, MM3) and semiempirical (AM1) calculations predicted structure 1c to be most stable followed by 1e, higher level ab initio methods
DZSCF and DZPSCF, with and without correlation at the MP2 level, predicted structure 1e to be the most stable, followed by structure 1c. In a more recent study, ${ }^{\text {aa }}$ Sulzbach et al. found a nearly planar "mono-trans" configuration of symmetry $C_{s}$ (1f) to be the most stable [10]annulene isomer at the MP2(fc)/DZd and higher levels, as well as the B3LYP density functional theory level. Depending on the level of calculation, it was found to be from 4 to $9 \mathrm{kcal} / \mathrm{mol}$ lower in energy than 1e. These authors could not locate structure $1 \mathrm{f}$ by the random search procedure employing the MM3 force field and claimed that because this structure was not a minimum at any but correlated levels, it could not be located by MM3. However, by constructing an initial mono-trans planar structure, we did find a stable MM3 structure of $C_{s}$ symmetry, although the energy was 37 and $27 \mathrm{kcal}$ higher than that of $1 \mathrm{c}$ before and after correlation energy was added, respectively, making it the highest in energy among the four stable structures considered in this work. The MM3 structure is remarkably similar to that from the highest level ab initio calculation by Sulzbach et al. ${ }^{9 a}$ The bond lengths are compared in Figure 1f. (Note that the MM3 bond lengths are $r_{g}$ while those from the B3LYP method should be $r_{e}$, but contain whatever systematic error is characteristic of the method. It would appear that this systematic error is close to the $r_{e} \rightarrow r_{g}$ correction.) Furthermore, we also found the inner $\mathrm{C}-\mathrm{H}$ bond to be shorter by $0.01 \AA$ than the other $\mathrm{C}-\mathrm{H}$ bonds in this structure, and that this bond is bent out of the approximate carbon plane by $26^{\circ}$, which is slightly larger than the $a b$ initio value of $20^{\circ}$. The energies of the four mini- 
TABLE II.

Energies (kcal / mol) of Four Stable Conformers of [10]Annulene Predicted by MM3.

\begin{tabular}{|c|c|c|c|c|}
\hline & \multicolumn{4}{|c|}{ Structure } \\
\hline & $\begin{array}{l}\mathrm{C}_{2 h} \\
\mathbf{1 b}\end{array}$ & $\begin{array}{l}\mathrm{C}_{2} \\
\mathbf{1 c}\end{array}$ & $\begin{array}{l}\mathrm{C}_{2} \\
1 \mathbf{e}\end{array}$ & $\begin{array}{l}\mathrm{C}_{\mathrm{s}} \\
\mathbf{1 f}\end{array}$ \\
\hline Steric energy & 51.3 & 43.9 & 46.7 & 78.9 \\
\hline Compression & 1.25 & 1.05 & 0.76 & 1.52 \\
\hline Bending & 3.93 & 3.56 & 2.17 & 51.73 \\
\hline Bend-bend & 0.12 & 0.11 & 0.07 & 3.04 \\
\hline Stretch-bend & 0.12 & 0.09 & 0.05 & 0.82 \\
\hline van der Waals, $1-4$ & 5.54 & 5.29 & 5.23 & 9.31 \\
\hline van der Waals, other & 9.44 & 7.42 & 3.91 & 4.11 \\
\hline Torsional & 25.76 & 20.15 & 26.98 & 0.70 \\
\hline Dipole-dipole & 6.16 & 6.21 & 7.54 & 7.67 \\
\hline Total MM3 energy (inc. HF) & -1080.7 & -1087.9 & -1084.1 & -1050.9 \\
\hline Correlation energy MP2 $* 2$ & -43.2 & -35.0 & -35.6 & -45.2 \\
\hline \multicolumn{5}{|l|}{ Relative energy } \\
\hline Without correlation & 7.2 & 0.0 & 3.8 & 37.0 \\
\hline With correlation & 0.0 & 1.0 & 4.2 & 27.8 \\
\hline
\end{tabular}

mum energy structures from MM3 are compared in Table II.

In a subsequent article, Sulzbach et al. ${ }^{9 b}$ reported single point energy calculations for the MP2/DZd and B3LYP/DZd optimized geometries of $1 \mathrm{e}$ and $\mathbf{1 f}$ with the high-level CCSD, CCSD(T), and UNO-CAS methods. The results now showed the energy of the twisted form 1e to be lower than that of 1 f by 3-7 $\mathrm{kcal} / \mathrm{mol}$. (It should be noted that structure $\mathbf{1 b}$ was not reported by any of the investigators mentioned above; therefore, it is not clear how it would compare in energy with the other structures if subjected to the same level of $a b$ initio calculations.)

The MM3 structures of $\mathbf{1 e}$ and $\mathbf{1 f}$ are very similar to those reported by Sulzbach et al. ${ }^{9 b}$ We were consequently surprised that the relative energies of 1e and 1f were so different in the two calculations. The MM3 energy for If appears to be much too high relative to $1 \mathrm{e}$ (and $\mathbf{1 c}$ and $\mathbf{1 b}$ ). But the $\pi$ system in 1f appears unexceptional, and we would expect MM3 to deal with it adequately (although the $a b$ initio work suggests that the MP2 level of correlation may lead to more than the usual error in this case). The only other large deformation in 1f is bending, and MM3 deals well with that. Thus, we expect the absolute energy (heat of formation) of $\mathbf{1 f}$ to be reasonable.

On the other hand, $\mathbf{1 b}, \mathbf{1 c}$, and $\mathbf{1 e}$ are all nonplanar. In developing MM4, the torsion barriers about carbon-carbon double bonds were increased about
$50 \%$ relative to those in MM3. The MM4 values were still lower than were required to fit the experiment, but they were a compromise chosen in an effort to fit other data. ${ }^{10}$ Because we were not prepared to extend the present work to MM4 at this time, we chose instead to estimate the importance of these torsional barrier errors in the present problem as follows. The relative energies of the four structures of interest are given in Table III, first as calculated by MM3 (with correlation), and second as calculated with a $50 \%$ increase in the torsional barriers about the carbon-carbon double bonds. (Some of the $a b$ initio results mentioned earlier are also included in Table III.)

Note that 1c now is somewhat more stable than $\mathbf{1 b}$, and the energy of $\mathbf{1 f}$ has dropped greatly from 27.8 to $15.3 \mathrm{kcal} / \mathrm{mol}$. These are estimated values, because we are not now prepared to really carry out this calculation. However, we now believe that we understand the source of most of the discrepancies between the $a b$ initio and MM3 calculations.

Our current "best" MM3 results are shown in line 2 of Table III, including a 50\% torsion error correction. This correction may be insufficient. If so, increasing it would raise the energies of $\mathbf{1 b}$ and 1e similarly and would raise 1c somewhat less $(80 \%)$, and 1f would not change. Thus, 1c would remain lowest in energy, with $\mathbf{1 b}$ slightly higher.

Our conclusion from the MM3 is that 1c, or possibly $\mathbf{1 b}$, should be the most stable; however, 


\begin{tabular}{|c|c|c|c|c|}
\hline & \multicolumn{4}{|c|}{ Structure } \\
\hline & $\begin{array}{c}\text { 1b } \\
\mathrm{C}_{2 \mathrm{~h}} \text { Nonpl. }\end{array}$ & $\begin{array}{c}1 \mathbf{1 c} \\
\mathrm{C}_{2} \text { Nonpl. }\end{array}$ & $\begin{array}{c}1 \mathbf{e} \\
\mathrm{C}_{2} \text { Nonpl. }\end{array}$ & $\begin{array}{c}1 f \\
\mathrm{C}_{\mathrm{s}} \mathrm{Pl}\end{array}$ \\
\hline $\begin{array}{l}\text { MM3 + correl. } \\
\text { (from Table II) }\end{array}$ & 0.0 & 1.0 & 4.2 & 27.8 \\
\hline $\begin{array}{l}\text { With } 50 \% \\
\text { Incr. torsion }\end{array}$ & 1.8 & 0.0 & 6.6 & 15.3 \\
\hline Ab initio ${ }^{\mathrm{a}}$ & NS & 0.0 & $11.9-13.8$ & NS \\
\hline$A b$ initio $^{b}$ & NS & $0.5-3.0$ & 0.0 & NS \\
\hline$A b$ initio $^{c}$ & NS & NS & $4-9$ & 0.0 \\
\hline$A b$ initio $^{d}$ & NS & NS & 0.0 & $3-7$ \\
\hline
\end{tabular}

NS, not studied.

${ }^{a}$ See text, ref. 8.

${ }^{\mathrm{b}}$ See text, ref. 4.

${ }^{\mathrm{c}}$ See text, ref. $9 \mathrm{a}$.

${ }^{\mathrm{d}}$ See text, ref. 9b.

there is much uncertainty here. In our view, the $a b$ initio results so far reported are too fragmentary to draw firm conclusions.

\section{[18]ANNULENE}

Earlier MM calculations using only the blockdiagonal minimization procedure reported three stable structures for this molecule: $\mathrm{D}_{6 \mathrm{~h}}, \mathrm{D}_{3 \mathrm{~h}}$, and $\mathrm{D}_{3}$. Our MM3 calculation employing the full-matrix method revealed three imaginary frequencies for the $D_{3 h}$ structure, indicating it to be at a stationary point but not at a local minimum. The experimental (X-ray) structure ${ }^{11}$ and three structures calculated by MM3 are summarized in Table IV. Also included are the relative energies obtained by MM1, ${ }^{12} \mathrm{MM} 2{ }^{13}$ and MM3 with and without electron correlation.

The data in the table show that the $\mathrm{D}_{3}$ form has the lowest MM3 energy before correlation energy is included, but that the $\mathrm{D}_{6 \mathrm{~h}}$ becomes more stable after electron correlation energy is added. The difference in correlation energy is more than $30 \mathrm{kcal}$ in favor off the delocalized structure, $\mathrm{D}_{6 \mathrm{~h}}$, compared to $8 \mathrm{kcal}$ in the smaller [10]annulene. The $\mathrm{D}_{6 \mathrm{~h}}$ structure is in agreement with the $\mathrm{X}$-ray structure (Table IV).

Attempts to find a stationary point corresponding to a nonplanar $\mathrm{D}_{6}$ structure with $\mathrm{MM} 3$ were not successful.

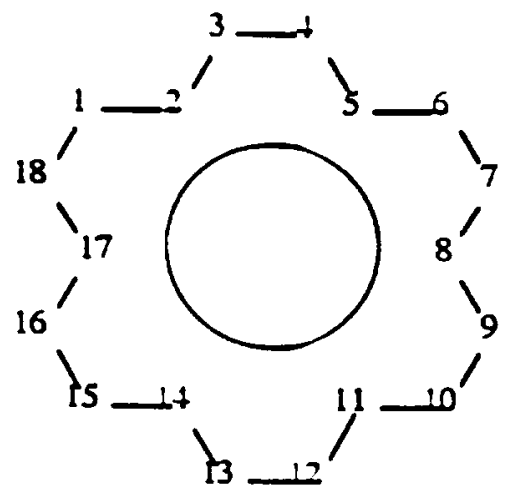

[18]annulene, $D_{6 h}$

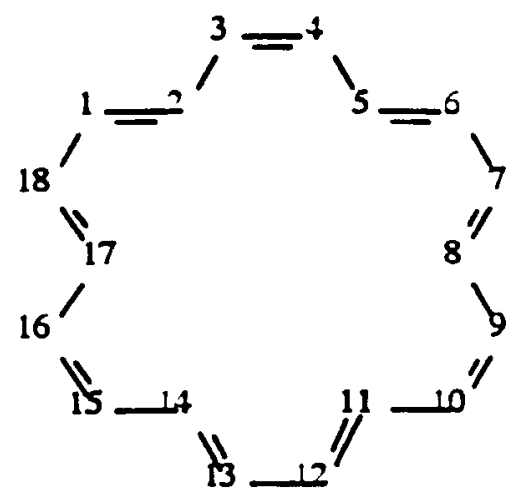

[18]annulene, $D_{3 b}$ or $D_{3}$ 
TABLE IV.

Bond Lengths (Å) and Energies (kcal / mol) of [18]Annulene Conformers.

\begin{tabular}{|c|c|c|c|c|}
\hline & \multicolumn{4}{|c|}{ Structure } \\
\hline & $\mathrm{D}_{6 \mathrm{~h}}$ & $\mathrm{D}_{3 \mathrm{~h}}{ }^{\mathrm{a}}$ & $\mathrm{D}_{3}$ & $\mathrm{X}$ ray $\left(\mathrm{D}_{6 \mathrm{~h}}\right)^{\mathrm{b}}$ \\
\hline 1-2 (inner or trans) & 1.406 & 1.358 & 1.357 & $1.382_{\text {ave }}$ \\
\hline 2-3 (inner) & 1.406 & 1.465 & 1.465 & 1.382 ave \\
\hline 3-4 (outer or cis) & 1.414 & 1.365 & 1.362 & 1.419 \\
\hline $4-5$ (inner) & & 1.465 & 1.465 & \\
\hline 5-6 (inner) & & 1.358 & 1.357 & \\
\hline $6-7$ (outer) & & 1.475 & 1.473 & \\
\hline \multicolumn{5}{|l|}{ Relative energy } \\
\hline MM1 & 10.2 & 8.9 & 0.0 & \\
\hline MM2 & 17.0 & 2.3 & 0.0 & \\
\hline MM3 & 25.8 & 0.6 & 0.0 & \\
\hline MM3 corr. energy & -99.6 & -61.5 & -62.1 & \\
\hline Relative energy $\mathrm{MM} 3$ with correlation & 0.0 & 12.8 & 11.6 & \\
\hline
\end{tabular}

Bond lengths are from simple MM3 calculations.

${ }^{\text {a }}$ Found to not be a stable structure by MM3.

${ }^{\mathrm{b}}$ Reference 11. Note that the X-ray bond lengths are $r_{\alpha}$ and were determined at room temperature. The MM3 bond lengths are $r_{\mathrm{g}}$ values. The interconversions of $r_{\alpha}, r_{\mathrm{g}}, r_{\mathrm{e}}, r_{\mathrm{z}}$, etc., have all been worked out and are included in MM3(96) (see ref. 14). The rigid body thermal motion correction and the sum of the other corrections would be expected to cause the X-ray bond lengths to increase by maybe $0.01 \AA$ or more, but corrections are not made here because of the preliminary nature of the MM3 bond lengths.

\section{BICYCLO[5.3.1]UNDECAPENTAENE}

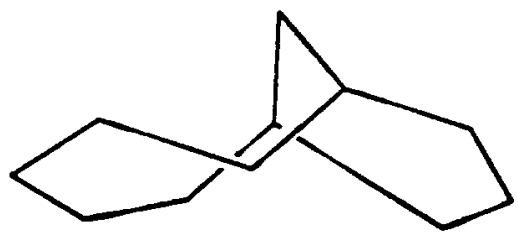

MM2 found the structure of this compound to be of alternate long and short bonds with no symmetry, ${ }^{13}$ while there was experimental evidence ${ }^{15}$ that the bonds should be more or less equal as in azulene. We found with MM3 a structure similar to that from MM2 (Fig. 2). After some search we found another stable structure (Fig. 3). It has $C_{s}$ symmetry, a higher steric energy, and a higher Hartree-Fock energy, but a more negative correlated energy than the $C_{1}$ structure. The $C_{s}$ structure shows nonalternate bond lengths and more planarity than the $\mathrm{C}_{1}$ structure and is in good agreement with the $\mathrm{X}$-ray structure. The bond lengths and the dihedral angles of both structures calculated at the MM3 level are given in Figures 2 and 3 below. The X-ray structure is also shown in Figure 3.

The calculated energies of the two conformers are given in Table V. It can be seen that before

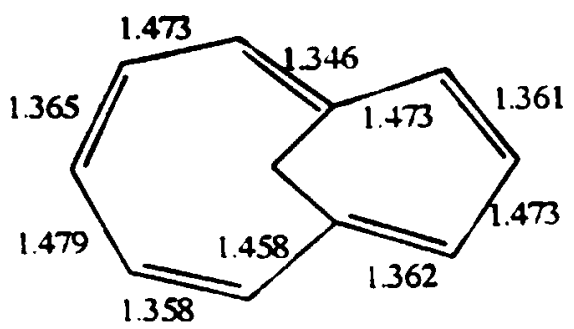

bond lengths

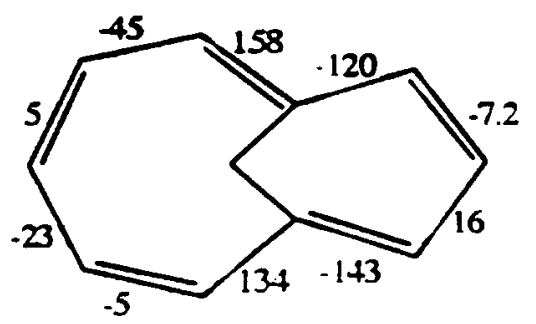

dihedral angles

FIGURE 2. The MM3 $C_{1}$ structure of bicyclo[5.3.1]undecapentaene.

electron correlation is included the $\mathrm{C}_{1}$ conformer is more stable by about $7 \mathrm{kcal} / \mathrm{mol}$, but after it is included the $\mathrm{C}_{\mathrm{s}}$ conformer becomes more stable by $1.3 \mathrm{kcal}$. Comparison of the $\mathrm{X}$-ray structure and the calculated $\mathrm{C}_{\mathrm{s}}$ structure shows good agreement. 


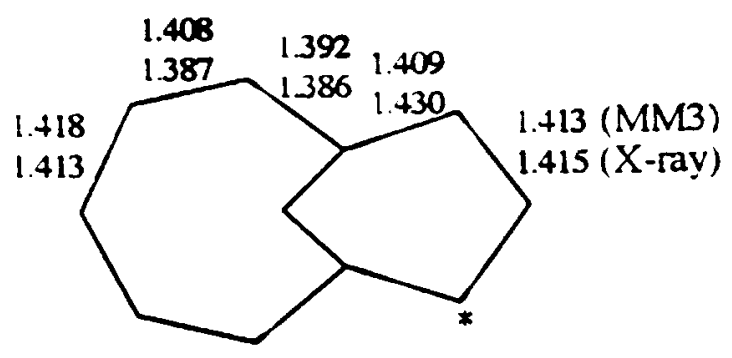

bond lengths

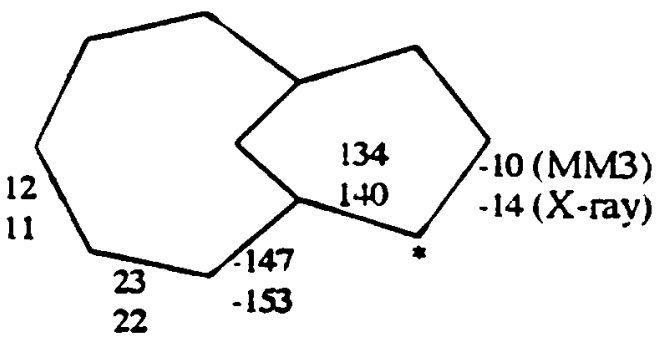

dihedral angles

FIGURE 3. The $\mathrm{C}_{\mathrm{s}}$ structure of

bicyclo[5.3.1] undecapentaene. The X-ray structure has a $-\mathrm{COCCl}_{3}$ group attached at the * position; see ref. 15.

\section{TABLE V.}

MM3 Energies (kcal/ mol) of Bicyclo[5.3.1]undecapentaene.

\begin{tabular}{lrr}
\hline & \multicolumn{2}{c}{ Structure } \\
\cline { 2 - 3 } & \multicolumn{1}{c}{$\mathrm{C}_{1}$} & \multicolumn{1}{c}{$\mathrm{C}_{\mathrm{s}}$} \\
\hline Steric energy & 47.5 & 54.9 \\
Total energy without correlation & -1082.6 & -1075.2 \\
Relative energy without & 0 & 7.4 \\
$\quad$ correlation & & \\
Correlation energy & -36.2 & -44.9 \\
Relative energy with correlation & 1.3 & 0.0 \\
\hline
\end{tabular}

\section{BICYCLO[4.4.1]UNDECAPENTAENE}

The NMR spectrum of bicyclo[4.4.1]undecapentaene showed eight protons at $\tau$ 7.5-6.8 and two protons at $\tau-0.5$, which may be considered as evidence of a ring current and suggests aromatic character and extensive conjugation. ${ }^{16}$ Furthermore, an X-ray examination of a crystalline carboxybicyclo[4.4.1]undecapentaene revealed a relatively planar structure with nonalternate outer ring bond lengths (excluding the bridge methylene

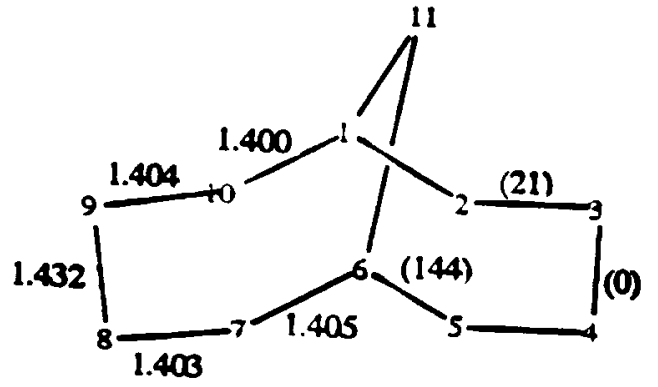

$4 a C_{2 v}$

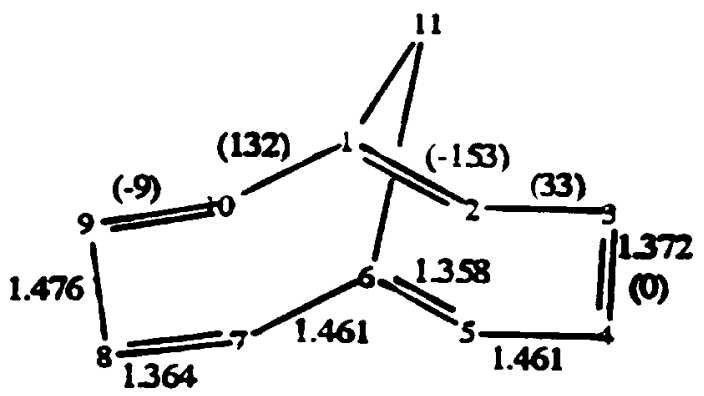

\section{$4 \mathrm{~b} \mathrm{C}_{3}$}

FIGURE 4. Bicyclo[4.4.1]undecapentaene. Numbers are bond lengths in angstroms, and numbers in parentheses are dihedral angles in degrees.

group). ${ }^{17}$ The MM2 article $^{13}$ reported a $C_{2 v}$ structure for this molecule with nonalternate bond lengths. The dihedral angles in the 10-membered ring were distorted no more than $30^{\circ}$ from planarity. The results were in good agreement with the X-ray structure. When an initial structure of this symmetry was minimized with MM3, the resultant structure was similar to what was calculated by MM2 (Fig. 4a). However, when the 3-4 bond was artificially shortened, the 8-9 bond was lengthened, and the structure was minimized again, a $\mathrm{C}_{\mathrm{s}}$ structure of lower energy resulted (Fig. $4 \mathrm{~b})$. The $\mathrm{C}_{\mathrm{s}}$ structure has alternate $\mathrm{C}-\mathrm{C}$ bonds. The mirror plane passes the middle of bonds 8-9 and 3-4 and atom 11. A search for a $C_{s}$ structure with a mirror plane passing through atoms 1,6 , and 11 was unsuccessful.

Correlated MM3 calculations were then carried out for the $C_{2 v}$ and $C_{s}$ structures. Again, the correlation energy favors the form with nonalternate bonds. The earlier MM2 work reported the correct structure $C_{2 v}$ by accident because it failed to find the $C_{s}$ structure, which would have the lower energy at the simple MM2 level of calcula- 
TABLE VI.

Energies ( $\mathrm{kcal} / \mathrm{mol})$ of Bicyclo[4.4.1]-

undecapentaene Calculated by MM3.

\begin{tabular}{lcr}
\hline & \multicolumn{2}{c}{ Structure } \\
\cline { 2 - 3 } & \multicolumn{1}{c}{$\mathrm{C}_{\mathrm{s}}$} & \multicolumn{1}{c}{$\mathrm{C}_{2 \mathrm{v}}$} \\
\hline Steric energy & 38.7 & 44.8 \\
Total energy without correlation & -1094.9 & -1090.0 \\
Relative energy without & 0 & 4.9 \\
$\quad$ correlation & & \\
Correlation energy & -35.8 & -44.0 \\
Relative energy with correlation & 3.3 & 0 \\
\hline
\end{tabular}

tion. The energies of the two forms are given in Table VI.

\section{PORPHIN}

The MM3 structure of porphin is of $C_{2 v}$ symmetry with one mirror plane passing through the middle of two of the pyrrole rings (Fig. 5a). ${ }^{18} \mathrm{An}$ X-ray study ${ }^{19}$ showed the molecule to possess approximate $\mathrm{D}_{2 \mathrm{~h}}$ symmetry (Fig. $5 \mathrm{~b}$ ) with nearly equal $\mathrm{C}-\mathrm{N}$ bonds and nearly equal bridge $\mathrm{C}-\mathrm{C}$ bonds. An $a b$ initio calculation ${ }^{3}$ found the $\mathrm{C}_{2 \mathrm{v}}$ form to have a lower energy at the Hartree-Fock level and the $D_{2 h}$ to have a lower energy after electron correlation was added. The magnitude of the correlation correction was dependent on the wave function used in the calculation.

Our attempts to find possible structures other than the $C_{2 v}$ were not successful. It appears that the MM3 formulation and the current parameters associated with the two nitrogen types would not allow an approximate $D_{2 h}$ symmetry. By significantly increasing the electron attracting power of the pyridine-type nitrogen atom and decreasing the electron attracting power of the pyrrole-type nitrogen atoms, we did find a nearly $\mathrm{D}_{2 \mathrm{~h}}$ structure. However, the parameters that were required and the electron density distribution that resulted were not in accordance with physical reality, and we decided to not pursue that possibility further. Therefore, a comparison could not be made between a localized and a delocalized structure for this molecule.

\section{BICYCLO[6.2.0|DECAPENTAENE}

Three Kekule structures can be written for the compound as in Figure 6.

MM2 calculations on this compound ${ }^{20}$ found structure A to be planar and the most stable, the

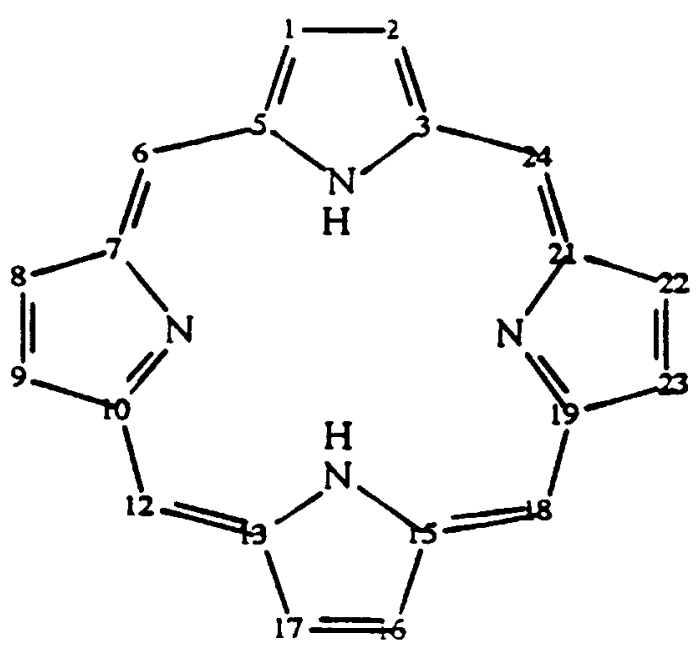

(a) $\mathbf{C}_{2 \mathbf{v}}$

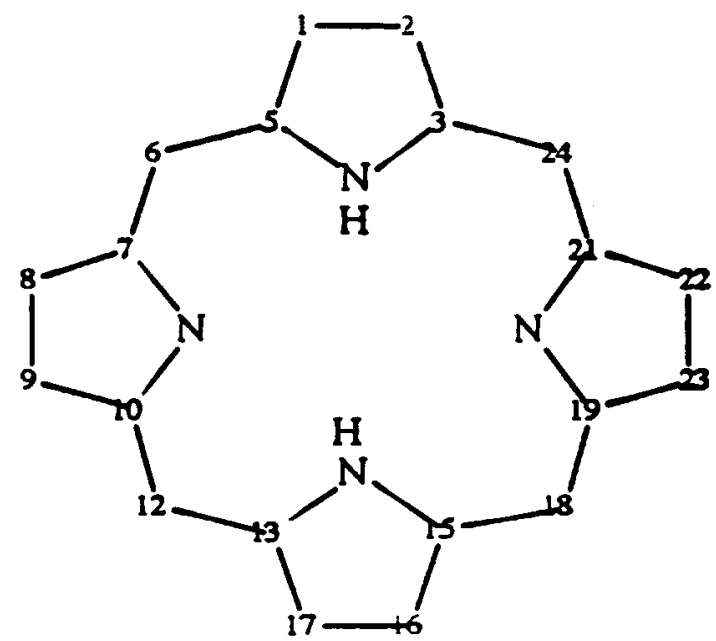

(b) $\mathbf{D}_{\mathbf{2 h}}$

FIGURE 5. Porphin.

planar form of structure B being $11 \mathrm{kcal}$ higher in energy. A nonplanar form of structure B was 3.7 kcal higher in energy than $A$, and structure $C$ was nonexistent. The cyclooctatetraene ring of the nonplanar B was found by MM2 calculation to be tub shaped with the dihedral angle 5-6-7-8 equal to $66^{\circ}$, and the cyclobutadiene ring was planar.

Our 1990 MM3 article, ${ }^{21}$ reported planar structure $\mathrm{A}$ to be the most stable followed by a shallow tub B, which was $15.9 \mathrm{kcal}$ higher in energy. The calculated structure A seems to agree fairly well with an X-ray analysis of the decapentaene nucleus of 9,10 diphenylbicyclo[6.2.0]decapentaene, ${ }^{22}$ which revealed near planarity of the decapentaene 
nucleus, a remarkably long central bond (1-2), and bond alternation of the peripheral bonds corresponding to structure A. The experimental deviations of the carbon atoms in the decapentaene nucleus from the best plane were found to be less than $0.150 \AA$, and the bond alternations were judged to be less than in a nonaromatic conjugated hydrocarbon. The calculated bond lengths showed a somewhat greater deviation.

A further MM3 study of this compound has now shown some unexpected results. While the planar structure $A$ is of the lowest energy among the three (A planar, B planar, and B nonplanar), the calculated vibration frequencies show an imaginary value indicating that the structure is not an energy minimum. Furthermore, the planar B is more stable than the nonplanar B structure, and although $C$ is not a stable structure, if the bonds 1-2 and 5-6 are restricted to $1.334 \AA$ and the rest of the molecule optimizd, the resultant structure has

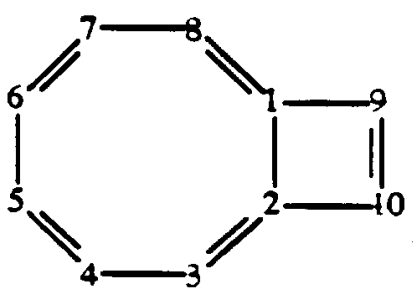

A

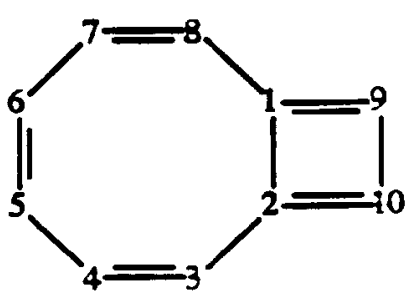

B

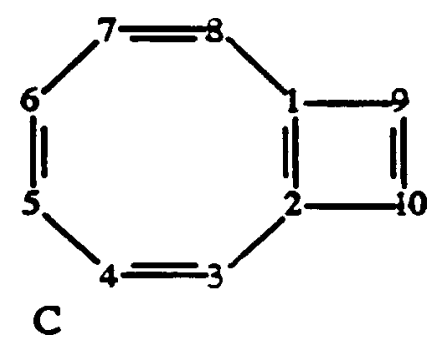

FIGURE 6. Kekule structures of bicyclo[6.2.0]decapentaene. an energy that is close to that of B. Inclusion of correlation does not change the relative energies of the four structures.

The fact that the MM3 structure for planar A is not a stable structure was not known from the earlier MM3 study, because when the study was carried out, the full-matrix minimization procedure was not an option in MM3 and vibrational frequencies were not calculated. After some search, a nonplanar form of A with an energy only 0.1 $\mathrm{kcal} / \mathrm{mol}$ lower than the planar A was found. This structure has $C_{2}$ symmetry, and the distances of the carbon atoms from the average plane range from 0.16 to $0.25 \AA$. The calculated bond lengths and the relative energies of the five structures are given in Table VII.

In order to be able to compare with experimental data more directly, we added the two phenyl groups to the planar decapentaene ring and repeated the calculation. Severe deviation from planarity occurred. The calculated structure had $\mathrm{C}_{2}$ symmetry and deviations of parent ring carbon atoms of up to $0.7 \AA$ from the average plane. The cyclooctatetraene ring puckered at about $38^{\circ}$. The phenyl groups were planar but rotated from the plane of the cyclobutene ring by about $35^{\circ}$. The shape and bond lengths of the decapentaene ring were qualitatively similar to those of structure A, except that in the parent compound, the deviation from planarity was much smaller. A comparison of the bond lengths with the experiment is given in Table VIII.

It is noted that the calculated bond alternation is greater than that observed experimentally. If the structure were to be minimized with respect to the total energy, including electron correlation energy, the calculated bond alternation would likely decrease, because our calculations on cyclic compounds have shown that electron correlation favors structures with less bond alternation. Because molecular mechanics minimizes with respect to steric energy, it could not automatically transit to a structure that is much lower in correlation energy, but higher in steric energy.

Because the energies between these forms do not differ very much for the isolated molecules and the crystal lattice may change the potential energy surface additionally, the molecules may be undergoing interchanges between these structures in the lattice. Given the relatively large experimental errors in the crystal structure, it is not clear just what is happening, nor is it clear how well the MM3 calculations reproduce the experiment. 
TABLE VII.

Comparison of Bond Lengths $(\AA)$ and Energies (kcal / mol) of Three Kekule Structures of Bicyclo[6.2.0]decapentaene.

\begin{tabular}{lccccc}
\hline Bond Length & A Planar $^{\mathrm{a}}$ & A Nonplanar & B Planar & B Nonplanar & C Planar $^{\mathrm{b}}$ \\
\hline $1-2$ & 1.510 & 1.501 & 1.587 & 1.581 & $1.334^{\mathrm{b}}$ \\
$2-3$ & 1.338 & 1.339 & & 1.443 & \\
$3-4$ & 1.454 & 1.462 & & 1.350 & \\
$4-5$ & 1.353 & 1.349 & & 1.464 & $1.334^{\mathrm{b}}$ \\
$5-6$ & 1.462 & 1.472 & 1.365 & 1.355 & \\
$1-9$ & 1.487 & 1.494 & & 1.547 & 89.4 \\
$9-10$ & 1.356 & 1.356 & 68.6 & 68.2 & 147.5 \\
$E_{s}$ & 67.0 & 130.3 & 146.2 & 147.1 & 17.2 \\
$\Delta H_{f}{ }^{c}$ & 130.4 & 0.0 & 15.9 & 16.8 & \\
$E_{\text {relative }}$ & 0.1 & & & & \\
\hline
\end{tabular}

${ }^{a}$ Not an energy minimum. However, the nonplanar A structures are lower in energy by only $0.1 \mathrm{kcal} /$ mol, which means that the lowest vibrational level is above this small saddlepoint. The molecule is predicted to be vibrating back and forth from structure A to its mirror image, and on average it is A planar.

${ }^{\mathrm{b}}$ Restricted.

${ }^{\mathrm{c}}$ Inclusion of correlation energy does not alter the relative energies of the energy minimum structures.

TABLE VIII.

Bond Lengths ( $(\AA)$ of 9,10-Diphenylbicyclo[6.2.0]decapentaene.

\begin{tabular}{lll}
\hline Bond Length & MM3 & Expt $^{\mathrm{a}}$ \\
\hline $1-2$ & 1.496 & 1.535 \\
$2-3$ & 1.341 & 1.336 \\
$3-4$ & 1.464 & 1.432 \\
$4-5$ & 1.348 & 1.374 \\
$5-6$ & 1.473 & 1.415 \\
$1-9$ & 1.498 & 1.446 \\
$9-10$ & 1.370 & 1.403 \\
$9-11$ & 1.472 & 1.461 \\
\hline
\end{tabular}

Average bond length, X-ray, $R=0.09$. Estimated standard deviation $=0.009-0.012 \AA$; probable error $\pm 0.02 \AA$.

${ }^{\text {a }}$ Reference 22.

\section{Conclusions} sions.

From this work, we made the following conclu-

1. As previously known, structure optimization starting with a certain symmetry often ends with the same symmetry, even if that is not the structure of lowest energy or even when that is not a minimum energy structure (see the case of [10]annulene). It is therefore important that different starting geometries be used in the conformational search when a compound has the potential of assuming geometries with different symmetries. The vibrational frequencies of the optimized structure must be examined to be sure that one is at an energy minimum.

2. In the context of MM3 calculations, an initial localized conjugated system will not convert to a delocalized one with the inclusion of electron correlation, even if the latter is more stable. One has to obtain the two structures separately, perfom a MP2 calculation on each, and compare their correlation energies. In cases such as the porphin molecule where MM3 does not locate two structures, no such comparison is possible. As a result, the experimental global minimum may not be found. No way is yet evident to automate the correlation calculations into MM3. Such calculations require much care on the part of the user, or misleading results are likely to be obtained.

We also confirm that for several examples

3. electron correlation is more important in structures with nonalternate (not necessarily equal) bond lengths and

4. the difference in correlation energy between the conformation with alternate single and double bonds and the conformation with nonalternate bonds becomes greater in larger annulenes. 
In view of the fact that MM3 works well for conjugated systems in the majority of cases, giving both geometries and energies (heats of formation), and because of conclusion 2 above, electron correlation will not be included in MM3 at this time. But it does seem clear that correlation at the MP2 level can greatly improve the calculated results in some cases, without an excessive expenditure of computer time.

Finally, it seems important to note that most of the errors in the MM3 calculations on conjugated hydrocarbons do not result from the semiempirical approximations used. Rather, they result from the Hartree-Fock approximation.

\section{Acknowledgment}

We are grateful to the Petroleum Research Fund of the American Chemical Society for support of this work.

\section{References}

1. The MM3 force field is described in J. Am. Chem. Soc. 111, $8551,8566,8576$ (1989), and in subsequent publications. The MM3 program is available from Tripos Associates (1699 South Hanley Road, St. Louis, MO 63144) to all users or from the Quantum Chemistry Program Exchange (Mr. Richard Counts, Indiana University, Bloomington, IN 47405) for academic users.

2. H. Baumann, J. Am. Chem. Soc. 100, 7196 (1978).

3. J. Almlöf, T. H. Fischer, P. G. Gassman, A. Ghosh, and M. Haser, J. Phys. Chem., 97, 10964 (1993).

4. Y. Xie, H. F. Schaefer III, G. Liang, and J. P. Bowen, J. Am. Chem. Soc., 116, 1442 (1994).

5. K. Yoshizawa, T. Kato, and T. Yamabe, J. Phys. Chem., 100, 5697 (1996).

6. (a) W. J. Hehre, L. Radom, P. v. R. Schleyer, and J. A. Pople, Ab Initio Molecular Orbital Theory, Wiley-Interscience, New York, 1986; (b) I. Levine, Quantum Chemistry, 3rd ed., Allyn and Bacon, Boston, 1983.

7. (a) U. Burkert and N. L. Allinger, Molecular Mechanics, American Chemical Society, Washington, D.C., 1982; (b) The block-diagonal minimization converges quickly and reliably when far from the minimum energy structure, but the full-matrix minimization converges more rapidly near the minimum. It is advantageous to use the former minimization method with a crude starting geometry, followed by the latter when near the minimum. However, either method may end up at a saddlepoint or even a hilltop in the potential surface. Whether the structure corresponds to a minimum, a saddlepoint, or a hilltop can be determined from the calculated vibrational frequencies. If they are all real, the molecule is at an energy minimum, whereas imaginary frequencies indicate otherwise. This information is only available from the full-matrix minimization. In MM2 and earlier versions of MM3, the full-matrix minimization was not incorporated, and occasionally structures that were reported as local minimum structures in earlier articles were later found to be saddlepoint or hilltop structures.

8. L. Farnell, J. Kao, L. Radom, and H. F. Schaefer III, J. Am. Chem. Soc., 103, 2147 (1981).

9. (a) H. M. Sulzbach, P. v. R. Schleyer, H. Jiao, Y. Xie, and H. F. Schaefer III, J. Am. Chem. Soc., 117, 1369 (1995); (b) H. M. Sulzbach, H. F. Schaefer III, W. Klopper, and H. P. Luthi, J. Am. Chem. Soc., 118, 3519 (1996).

10. (a) N. Nevins, K.-H. Chen, and N. L. Allinger, J. Comput. Chem., 17, 669 (1996); (b) N. Nevins, J.-H. Lii, and N. L. Allinger, J. Comput. Chem., 17, 695 (1996).

11. J. Bergman, C. L. Hirshfeld, D. Rabinovich, and G. M. J. Schmidt, Acta Crystallogr., 19, 227 (1965).

12. N. L. Allinger and J. T. Sprague, J. Am. Chem. Soc., 95, 3893 (1973).

13. J. T. Sprague, J. C. Tai, Y. Yuh, and N. L. Allinger, J. Comput. Chem., 8, 581 (1987).

14. (a) B. Ma, J.-H. Lii, H. F. Schaefer III, and N. L. Allinger, J. Phys. Chem., 100, 8763 (1996); (b) B. Ma and N. L. Allinger, J. Mol. Struct., 413-414, 395 (1997); (c) B. Ma, J.-H. Lii, K. Chen, and N. L. Allinger, J. Am. Chem. Soc., 119, 2570 (1997).

15. L. T. Scott, M. Oda, and I. Erden, J. Am. Chem. Soc., 107, 7213 (1985).

16. E. Vogel and H. D. Roth, Angew. Chem. Int. Ed. Engl., 3, 228 (1964).

17. M. Dobler and J. D. Dunitz, Helv. Chim. Acta, 48, 1429 (1965).

18. J. C. Tai, L. Yang, and N. L. Allinger, J. Am. Chem. Soc., 115, 11906 (1993).

19. B. M. L. Chen and A. Tulinsky, J. Am. Chem. Soc., 94, 4144 (1972).

20. N. L. Allinger and Y. H. Yuh, Pure Appl. Chem., 55, 191 (1983).

21. N. L. Allinger, F. Li, L. Yan, and J. C. Tai, J. Comput. Chem., 11, 868 (1990).

22. C. Kabuto and M. Oda, Tetrahedron Lett., 21, 103 (1980). 